\title{
The nucleus as a fluid of skyrmions: Energy levels and nucleon properties in the medium*
}

\author{
G. Kälbermann \\ Faculty of Agriculture and Racah Institute of Physics \\ Hebrew University, Rehovot 76100, Israel
}

August 24, 2018

\begin{abstract}
A model of a fluid of skyrmions coupled to a scalar and to the $\omega$ meson mean fields is developed. The central and spin-orbit potentials of a skyrmion generated by the fields predict correct energy levels in selected closed shell nuclei. The effect of the meson fields on the properties of skyrmions in nuclei is investigated.
\end{abstract}

PACS 12.39Dc, 21.10Dr, 21.65.+f, 24.10.Pa

*dedicated to the memory of Prof. Judah M. Eisenberg 


\section{Introduction: Fluid models of nuclei}

Fluid models of nuclei have been used for quite a long time. The time-honored liquid drop model[1] is extremely successful in describing the ground state bulk properties of nuclei by utilizing extremely simple assumptions. These assumptions are based on the observation of the saturation phenomenon in

nuclei as the predominant effect in determining the binding energies. The analogy of the nucleus to a fluid is also borne out in the almost constant density of nuclei throughout the periodic table and the lack of localization of nucleons around fixed positions, as it would be for a solid, due to zero point motion. The nucleus is a quantum fluid and cannot be modeled as a usual fluid for which the molecules behave to a large extent classically.

More recently, the need to include relativistic effects in the description of hadron dynamics inside the nucleus lead to the development of the nuclear mean field models [2]. These theories can be viewed as fluid models in which the mean field plays the role of the continuum (hydrodynamical regime), while the elementary fermion fields are their microscopic source. Again, the model has proven fruitful in the prediction of ground states as well as collective states of nuclei.

On the other hand, nuclei -especially light ones- possess shell structures that are usually traced back to the single particle energy levels. This is clearly seen in the oscillations of nuclear binding energies as a function of mass, as well as in the existence or inexistence of stable nuclides.

Heavy nuclei, however, seem to behave as deformed rigid bodies. This collective behavior is put in evidence by the large quadrupole excitation amplitudes.

The question of the nature of the state of matter in the nucleus consequently depends on both the excitation energy, the mass and the density of the nucleus.

In any event, the nucleus cannot be compared to a solid. Even heavy 
nuclei have a rather soft incompressibility factor. Being compressible, they resemble more a gas or a compressible liquid, as advocated by the propugnators of the liquid drop model.

If one agrees with this view of a nucleus as a fluid, liquid or gas depending on the temperature, then it is valid to consider its description in terms of a few degrees of freedom as it is done for conventional fluids. The quantum nature of the nucleons demands the treatment to be quantal. The high speeds attainable by nucleons in the nucleus requires a relativistic treatment.

One key ingredient that is absent in all the hydrodynamical models is the nucleon finite size. The charge radius of the proton is not negligible compared to nuclear dimensions, in contradistinction to molecules in a liquid. In the nonrelativistic Hartree-Fock or relativistic mean field Hartree treatments of nuclei, the nucleon is treated as pointlike. Some information about the finite extent of the nucleon is sometimes included in the form of short range correlations, short range hard-core, etc.

The purpose of the present work is to try to deal in a coherent manner with the fluid aspect of nuclei and the finite size of the nucleon.

Much has been learned in the past 40 years about the nucleon. Despite the fact that QCD is the accepted theory of strong interactions, it is practically impossible to use it as a tool to generate baryons as confined objects of quarks and gluons, and even less to describe nuclei. We here resort to a low energy model of strong interactions, namely, the Skyrme model[3]. The issue of finite nucleon size is dealt with by describing the nucleon as a topological soliton, the skyrmion. The fluid aspect is addressed by using a relativistic mean field theory in the spirit of the Walecka model[2]. The scheme is simplified by resorting to a dilute fluid approximation to be explained below. In this manner the nucleons are essentially free, interactions being mediated by the mean fields. Section 2 summarizes the formalism and previous results both for nuclear matter and finite nuclei. Section 3 is devoted to the energy levels of a skyrmion in the nucleus. Section 4 treats the effects of the meson fields 
on the properties of the skyrmion.

\section{A skyrmion fluid model of nuclei}

In the early 60's Skyrme developed a topological soliton model for nucleons [3]. In this model, baryons emerge as classical topological solutions of a nonlinear meson lagrangian. More than a decade ago this perspective was taken up by Witten [4]. He showed that baryons may indeed appear as solitons in the large $N_{c}$ limit of QCD. The development of collective coordinate quantization of soliton rotations [5] gave a tremendous boost to the field. The door was now open for the investigation of baryons and nuclei. The Skyrme model and its topological solitons: skyrmions, may be regarded as the effective degrees of freedom of low energy baryon physics. This is especially appealing due to the difficulty of solving QCD explicitly.

The Skyrme model has had moderate success in dealing with the nucleonnucleon interaction, including the attractive isoscalar central potential for which several mechanisms were proposed [6]. It is difficult to solve the model exactly for large baryon number structures. Static solutions of various geometries for some special baryon number cases were recently found using the rational map ansatz[7]. These solutions are of a limited practical use for the treatment of nuclei. Nuclei appear to be extremely dynamical assembly of nucleons. The special static solutions found in the literature resemble a crystal[8]. Some of these solutions are in conflict with the spin-isospin assignment of nuclear ground states[9]. We here opt for an approach in which

the nucleus is viewed as a dynamical aggregate of nucleons. For this purpose it is convenient to follow the path of fluid-like mean field theories, such as the relativistic model of Walecka[2].

In the relativistic mean field theory of Walecka the nucleons are described using Dirac wave functions. The nucleons interact with meson fields. The meson fields are in turn determined by the various densities of the 
baryons(scalar, vector,etc.). The nucleons have no internal structure. They are considered elementary pointlike objects. The model is simple enough to handle the basic features of nuclei and nuclear matter, and yet powerful enough to predict ground states and excited states with a relatively small amount of parameters that enter in the meson self-interactions and mesonnucleon coupling constants. The model is used at the first quantized level. Quantum corrections spoil the predictions. Being only a low energy effective model for nuclei, it is valid to consider it within its realm of applicability: the mean field level.

The same path will be followed here. We will construct and use a mean field model of nucleons in terms of skyrmions, instead of Dirac pointlike objects. The task becomes more complicated due to the finite volume occupied by the skyrmion. In some sense the Dirac wave function represents only the center of mass dynamics, while, the first quantized skyrmion will carry information about the interior of the nucleon.

The skyrmion lagrangian is built by demanding both isospin and chiral symmetries $(\mathrm{SU}(2) \mathrm{xSU}(2))$. The many-body model developed here still obeys the same principles.

The mean fields considered in the present work are the minimal set in order to be able to describe low energy nuclear phenomenology. A scalar field provides the attractive interaction and binds nuclei. The coupling of the scalar to the soliton is taken from phenomelogically succesful models 10 , [1], 12]. The added scalar brings about the intermediate range attraction between skyrmions 13, that is absent for potentials generated from the product ansatz.

In order to stabilize the nucleus and reproduce the saturation property we also need a repulsive interaction. We here follow the Walecka models and use a meson field that is an isoscalar-vector field, the $\omega$ meson.

Consider a field theory lagrangian of skyrmions, a scalar $\psi$ and the $\omega$ 
meson 6]

$$
\begin{aligned}
\mathcal{L} & =\mathcal{L}_{2} \psi+\mathcal{L}_{2}+\mathcal{L}_{4}-V_{\text {interaction }}-V(\psi)+\mathcal{L}_{\omega} \\
& =\frac{1}{2} \partial_{\mu} \psi \partial^{\mu} \psi-g_{\psi} \frac{\psi}{\gamma} \frac{F_{\pi}^{2}}{16} \operatorname{tr}\left(L_{\mu} L^{\mu}\right)+\frac{1}{32 e^{2}} \operatorname{tr}\left[L_{\mu}, L_{\nu}\right]^{2}-g_{V} \omega_{\mu} B^{\mu} \\
& -V(\psi)-\frac{1}{4}\left(\partial_{\mu} \omega_{\nu}-\partial_{\nu} \omega_{\mu}\right)^{2}+\frac{\psi^{2}}{2 \gamma^{2}} m_{\omega}^{2} \omega_{\mu}^{2}
\end{aligned}
$$

Here

$$
L_{\mu} \equiv U^{\dagger} \partial_{\mu} U
$$

where $U(\mathbf{r}, t)$ is the chiral field, $F_{\pi}$ is the pion decay constant and $e$ the Skyrme parameter, $\psi$ is the scalar, $\gamma$, an energy scale-parameter, and, $g_{\psi}$, a coupling constant. We have omitted the $\rho$ meson as it is our intention to treat symmetric nuclei only.

In the present work we will not specify the potential of the scalar. It must contain a mass-term and higher order terms.

Due to the fact that the sign of $\psi$ is irrelevant, it leads to an attractive interaction for both positive and negative signs, we can redefine the field in the form $\psi=\gamma e^{\sigma}$. We will henceforth absorb the coupling constant $g_{\psi}$ in the definition of the constant $F_{\pi}$, that is in any event a fitted parameter in the Skyrme model.

Similarly to the mean field theory of pointlike baryons, we consider an ensemble of essentially free skyrmions. Each skyrmion will be accompanied by its own scalar field and $\omega$ fields. Although we deal with free skyrmions, the average properties of the ensemble are still included in distribution functions that depend on the density and temperature.

The skyrmion is a topological soliton. The winding number of the soliton was already identified by Skyrme with the baryon charge. It is absolutely conserved regardless of the dynamics. It is a geometrical property, not a Noether charge. We have to take care of this aspect when building many 
body ansatze for the nucleon fluid. The baryon number should be exactly conserved.

An ansatz that conserves baryon number exactly and still allows for a reasonable treatment of the dynamics of $N$ skyrmions is the product ansatz

$$
U_{B=N}\left(\mathbf{r}, \mathbf{R}_{1}, \mathbf{R}_{2}, \cdots, \mathbf{R}_{N}\right)=U\left(\mathbf{r}-\mathbf{R}_{1}\right) U\left(\mathbf{r}-\mathbf{R}_{2}\right) \cdots U\left(\mathbf{r}-\mathbf{R}_{N}\right),
$$

Where $R_{i}$ is the location of the center of skyrmion $i$.

This ansatz is not an exact solution of the skyrmion sector of the problem. We mentioned static exact solutions [7] as being appropriate for a crystal-like structure of nuclei. It is our intention to treat the dynamics of the nucleons in the nucleus in a reasonable manner without sacrificing nucleon motion, hence we opt for this crude approximation. Despite being a rough ansatz, it is very maleable and quite appropriate for the nuclear case in which the nucleons are not tightly packed. Nucleons in the nucleus are separated by an average distance in the order of $2 \mathrm{fm}$. The solitons overlap at the 'surface' of each skyrmion. This interaction is replaced by the effective meson fields. Instead of dealing with the complicated situation of the N-body interaction in all its aspects, a small set of mesonic degrees of freedom is chosen and the skyrmions interact solely with them. It is expected that the introduction of the mesons in the mean fields will compensate, at a phenomenological level, the lack of accuracy induced by the rudimentary product ansatz.

For the meson fields we use (see section 4 for an improvement upon this approximation)

$$
\begin{aligned}
\sigma_{B=N} & =\sigma_{1}+\sigma_{2}+\cdots+\sigma_{N} \\
& =\sigma_{0}+\delta \sigma_{1}+\delta \sigma_{2}+\cdots+\delta \sigma_{N}, \\
\omega_{B=N} & =\omega_{1}+\omega_{2}+\cdots+\omega_{N} \\
& =\omega_{0}+\delta \omega_{1}+\delta \omega_{2}+\cdots+\delta \omega_{N},
\end{aligned}
$$


Where $\sigma_{0}, \omega_{0}$ are the mean field constant values of the scalar and the $\omega$ and $\delta \sigma, \delta \omega$ represent the fluctuations. In thermal equilibrium, the mean field fields will depend on the temperature $T$ and the chemical potential $\mu$. Self-consistency then demands the values of $\sigma_{0}$ and $\omega_{0}$ to be determined by the properties of the ensemble. In equilibrium the fluctuations will be taken to vanish in accordance with the mean field approximation.

The topological baryon density

$$
B^{\mu}=\frac{\epsilon^{\mu \alpha \beta \gamma}}{24 \pi^{2}} \operatorname{tr}\left[\left(U^{\dagger} \partial_{\alpha} U\right)\left(U^{\dagger} \partial_{\beta} U\right)\left(U^{\dagger} \partial_{\gamma} U\right)\right]
$$

with the the product ansatz of eq. (3) gives

$$
B_{0}=b_{1}+b_{2}+\cdots+b_{N}
$$

The dynamics of the nucleons is that of a rigid soliton. No internal excitations are considered. Also skyrmion distortions will not be allowed, except for swelling and shrinking of the soliton in the medium. Only the bulk parameters of the skyrmion, mass and moment of inertia will be allowed to vary due to the presence of the mesonic medium.

This is the basis for the dilute fluid approximation. It is related to the mean field Dirac model with two major modifications: 1) there is a different dynamics for the baryons dictated by the lagrangian of eq. (便) and, 2) there is a clear way to uncover the baryon response to the medium through the soliton equation of motion.

The many body skyrmion fluid in the dilute approximation is built from the product ansatz of eq. (3) for the skyrmions and the additive ansatz for the mesons of eq. (4). In the following we use as input a distribution function for the baryons as a shortcut.

A better method would be one that uses a fully self-consistent method, such as a Hartree-Fock. However, the use of a definite distribution function 
permits a first pass to the problem. We will use a Thomas-Fermi approximation: The skyrmion fluid is forced to remain within the boundaries of the nucleus. This introduces a cutoff Fermi radius. Beyond this radius the meson fields will still exist, but the baryon densities will be taken as vanishing.

We stress again that the results obtained here are to be taken within the context of the two approximations they hinge upon, namely, a simplistic product ansatz -dilute fluid approximation- and an averaging procedure with a pre-chosen distribution function. The former is a working hypothesis that permits the treatment of dynamics in a rather straightforward manner, and, the latter finds support in the the fact that we want to calculate equilibrium properties. The first approximation breaks down at short distances between nucleons and the second loses meaning for light nuclei, for which the 'meanfields' are not a wise choice of degrees of freedom, and direct nucleon-nucleon interactions are a better way to deal with the problem instead of by means of intermediaries.

The skyrmions dynamics is obtained by boosting the static skyrmions rigidly. We perform a Lorentz boost on the collective coordinate $R(t)$ of each skyrmion, although a non-relativistic Galilean transformation may suffice and perhaps be more consistent with the spirit of the rigid boosting. However, using a Lorentz transformation will help us to find the spin-orbit interaction below.

For the sake of simplicity consider a Lorentz boost along the $x$ axis with velocity parameter $v$ (For a different view of the boost problem see ref [14):

$$
\begin{aligned}
x \rightarrow \tilde{x} & =\frac{x-R(t)}{\sqrt{1-v^{2}}} \\
\tilde{y} & =y \\
\tilde{z} & =z \\
F(\overrightarrow{\mathbf{r}}) & \rightarrow F(\overrightarrow{\tilde{\mathbf{r}}})
\end{aligned}
$$




$$
\begin{aligned}
\sigma(\overrightarrow{\mathbf{r}}) & \rightarrow \sigma(\overrightarrow{\tilde{\mathbf{r}}}) \\
\omega^{\mu} & \rightarrow\left(\frac{\omega(\overrightarrow{\overrightarrow{\mathbf{r}}})}{\sqrt{1-v^{2}}}, \frac{v \omega(\overrightarrow{\tilde{\mathbf{r}}})}{\sqrt{1-v^{2}}}, 0,0\right) \\
B^{\mu} & \rightarrow\left(\frac{B_{0}(\overrightarrow{\overrightarrow{\mathbf{r}}})}{\sqrt{1-v^{2}}}, \frac{v B_{0}(\overrightarrow{\tilde{\mathbf{r}}})}{\sqrt{1-v^{2}}}, 0,0\right)
\end{aligned}
$$

with $\omega^{\mu} B_{\mu}=\omega_{0}(\overrightarrow{\tilde{\mathbf{r}}}) B_{0}(\overrightarrow{\tilde{\mathbf{r}}})$.

Introducing the above transformation in eq. (1) and calculating the Hamiltonian, we find the energy of a skyrmion in motion to be

$$
E_{p}=\left(E_{2}+E_{\sigma}-E_{\omega}\right) \frac{2 p^{2}+3 M^{2}}{3 \epsilon M}+E_{4} \frac{4 p^{2}+3 M^{2}}{3 \epsilon M}+\frac{M}{\epsilon}\left(U_{\sigma}-U_{\omega}+U_{i n t}\right)
$$

where

$$
\epsilon=\sqrt{p^{2}+M^{2}}, \quad p=\frac{M v}{\sqrt{1-v^{2}}},
$$

$M$ is the skyrmion static mass for nonvanishing $\omega_{0}, \sigma_{0}$

$$
\begin{aligned}
& M(R)=4 \pi \int_{0}^{\infty} r^{2} d r M(r) \\
& M(r)=\mathrm{e}^{2 \sigma} \frac{F_{\pi}^{2}}{8}\left[F^{\prime 2}+2 \frac{\sin ^{2} F}{r^{2}}\right]+\frac{1}{2 e^{2}} \frac{\sin ^{2} F}{r^{2}}\left[\frac{\sin ^{2} F}{r^{2}}+2 F^{\prime 2}\right]
\end{aligned}
$$

and

$$
\begin{aligned}
E_{2} & =\frac{4 \pi F_{\pi}^{2}}{8} \int r^{2} d r e^{2 \sigma}\left(F^{2}+\frac{2 \sin ^{2} F}{r^{2}}\right) \\
E_{4} & =\frac{4 \pi}{2 e^{2}} \int d r\left(2 F^{2}+\frac{\sin ^{2} F}{r^{2}}\right) \\
E_{\sigma} & =\frac{4 \pi \Gamma_{0}^{2}}{2} \int r^{2} d r e^{2 \sigma} \sigma^{2} \\
E_{\omega} & =4 \pi \int r^{2} d r \omega^{\prime 2} \\
U_{\sigma} & =4 \pi \int r^{2} d r V_{\sigma} \\
U_{\omega} & =4 \pi \int r^{2} d r e^{2 \sigma} \frac{m_{\omega}^{2} \omega^{2}}{2} \\
U_{i n t} & =\frac{2 g_{V}}{\pi} \int d r \omega F^{\prime} \sin ^{2} F
\end{aligned}
$$


If the fields are kept as constant throughout the nucleon volume, we can use the skyrmion profile equation (or the virial theorem) to obtain

$$
M(R)=e^{\sigma} M_{0}
$$

where $M_{0}$ is the skyrmion mass for $\sigma=0$. We can include the rotational energy of the skyrmion in the adiabatic approximation. The result will yield the same scaling law as above. An attempt to include the rotational energy beyond the adiabatic approximation will induce the known instabilities to radiation of pions. Henceforth we use the measured free nucleon mass for $M_{0}$, that contains both static and rotational energies. The above scaling is the only effect of the mean fields on the skyrmion, while the skyrmion contributes to the mean fields through its energy and baryon number separately. The topological character of the baryon current in the Skyrme model prevents it from changing. The only effect of the mean fields on the skyrmion is that of mass scaling.

We can now write down the energy of $\mathrm{N}$ skyrmions in the mean field approximation with a Thomas-Fermi distribution

$$
f\left(\mathbf{p}_{i}, \mathbf{R}_{i}\right)=\Theta\left(p_{F}\left(R_{i}\right)-p_{i}\right)
$$

with the normalization condition

$$
\int d \mathbf{R}_{i} d \mathbf{p}_{i} f=1
$$

The Fermi momentum $p_{F}$ depends on the distance $R_{i}$ of the center of the $i^{\text {th }}$ skyrmion from the center of the nucleus. Also, for indistinguishable particles $p_{F}\left(R_{i}\right)=p_{F}(R)$. The distribution function for the ensemble is just a product of functions of the type above.

Averaging over the coordinates $R$ and the corresponding momenta with the distribution function for zero temperature, the energy of the nucleus with spherical symmetry we obtain, using eq.(9) 


$$
\begin{aligned}
E & =4 \pi \int R^{2} d R E(R) \\
E(R) & =E_{\sigma}+E_{\omega}+E_{\text {int }}+E_{s k}
\end{aligned}
$$

where

$$
\begin{aligned}
E_{\sigma} & =\frac{1}{2} \Gamma_{0}^{2} e^{2 \sigma} \sigma^{2}+V_{\sigma} \\
E_{\omega} & =-\frac{1}{2} \omega^{\prime 2}-e^{2 \sigma} \frac{m_{\omega}^{2} \omega^{2}}{2} \\
E_{\text {int }} & =g_{V} \omega B \\
E_{s k} & =\frac{1}{\pi^{2}} \int_{0}^{k_{P}} k^{2} d k \sqrt{k^{2}+M^{2}}+\frac{1}{\pi^{2}} \int_{0}^{k_{N}} k^{2} d k \sqrt{k^{2}+M^{2}}
\end{aligned}
$$

where $k_{P}, k_{N}$ are the proton and neutron local (R dependent) Fermi momenta.

In eq. (16) primes denote derivatives with respect to $R$, whereas in eq. (10) they represent derivatives with respect to $r$. Also

$$
B(R)=\frac{\left(k_{P}\right)^{3}}{3 \pi^{2}}+\frac{\left(k_{N}\right)^{3}}{3 \pi^{2}}
$$

The Euler-Lagrange equations for the mean fields become

$$
\begin{aligned}
\Gamma_{0}^{2} e^{2 \sigma}\left(\sigma^{\prime \prime}+2 \sigma^{\prime 2}+\frac{2 \sigma^{\prime}}{R}\right)-\frac{d V_{\sigma}}{d \sigma}+m_{\omega}^{2} \omega^{2} e^{2 \sigma}-\frac{\partial E_{s k}}{\partial \sigma} & =0 \\
\omega^{\prime \prime}+\frac{2 \omega^{\prime}}{R}-m_{\omega}^{2} \omega e^{2 \sigma}+g_{V} B & =0
\end{aligned}
$$

The ground state of the nucleus for fixed number of protons $(\mathrm{P})$ and neutrons $(\mathrm{N})$ is obtained by minimizing the energy, constrained by Lagrange multipliers, with respect to the local wavenumbers $k_{P}$ and $k_{N}$ 


$$
\delta E-\mu_{P} \delta P-\mu_{N} \delta N=0
$$

The algebraic equations for the multipliers become [2]

$$
\begin{aligned}
& \mu_{P}=g_{V} \omega+\frac{1}{2} g_{\rho} b+q a+\sqrt{k_{P}^{2}+M^{2}} \\
& \mu_{N}=g_{V} \omega-\frac{1}{2} g_{\rho} b+\sqrt{k_{N}^{2}+M^{2}}
\end{aligned}
$$

In the Thomas-Fermi approximation, the nucleus has a finite radius beyond which the nucleon densities are taken to vanish, therefore $\mu_{P}=\mu_{N}$.

In previous works [15, 16], we found that it is possible to fit the properties of nuclear matter, including the incompressibility, around $270 \mathrm{MeV}$, and the ground state densities of some magic nuclei, by introducing a parametrization of the scalar potential with 4 parameters.

The key restriction for the terms in the extended potential comes from the requirement that the expectation value of the $\sigma$ field in the vacuum remains unchanged $\langle v a c|\sigma| v a c\rangle=0$. Terms of the form $e^{n \sigma}-1$, generically referred to as no-log terms [11], are acceptable.

In the present work we will not commit ourselves as to the nature of the scalar field and its mass. We will not determine the scalar potential either. The field will be determined phenomenologically from the nuclear densities.

\section{$3 \quad$ Energy levels of a skyrmion in the nucleus}

In the present work we address the question of the energy levels and properties of the nucleon in the skyrmion fluid. To this end we simplify the treatment of section 2 . We solve the $\omega$ meson equation (18) with $B(R)$ replaced by the measured densities and the scalar determined by the chemical potential. In this manner we avoid tedious parametrizations of the scalar potential in order to fit the densities. The assumption is that such a potential 
exists, and the results of ref. [15] support it. The equation of the $\sigma$ meson is therefore not solved. The $\omega$ meson eq. (18) is solved starting at the center of the nucleus up to a cutoff radius $R_{c}$ at which $\omega=0$. The $\sigma$ field is then extrapolated beyond that point using suitable boundary conditions [2, 16].

A key element of the nucleon interaction in the nucleus is the spin-orbit potential. We will here proceed to consider the contribution of both the scalar and vector fields to this force. The dominant contribution will arise from the modification of the mean fields in a rotating frame as viewed by the moving nucleon. The treatment will be a mixture of relativistic (when essential) and nonrelativistic methods. A more coherent treatment would call for a parametrization of the metric of a rotating nucleus, but this is outside the scope of the present work.

Consider first a rigid boost of the skyrmion center with velocity $\mathbf{v}$ of eq. (8) together with a rigid rotation of the skyrmion with collective coordinates decribing spin and isospin 4 ]

$$
U(\tilde{\mathbf{r}}) \rightarrow A(t) U(\tilde{\mathbf{r}}) A^{\dagger}(t)
$$

The spin-orbit interaction may then arise from the well-known Thomas precession. It can be obtained from two consecutive Lorentz transformations. This spin-orbit force will exist regardless of the extended nature of the skyrmion provided it moves in a central force field and it carries an axis. An easy way to implement this transformation is to take the isospin vector matrix as time dependent

$$
\dot{\tau}=-\Omega_{T} \times \boldsymbol{\tau}
$$

where $\Omega_{T}$ is the Thomas frequency. Inserting the above ansatz in the skyrmion 
lagrangian of eq. (11) the spin-orbit interaction at the lowest order in the velocity is found to be

$$
U_{\text {s.o. }} \approx-\frac{\mathbf{S} \cdot \mathbf{L}}{2 M_{0}^{2} R} \frac{\partial V_{C}}{\partial R}
$$

where $V_{C}$ is the central potential of the skyrmion in the nucleus, $\mathbf{S}$ is the spin and $\mathbf{L}$ the angular momentum. The same result as found in standard textbook derivations [17]. In eq.(23) we have used the projection formula [4]

$$
\dot{A}^{\dagger} A=\frac{-i \boldsymbol{\tau} \cdot \mathbf{S}}{2 \lambda(R)}
$$

where $\lambda(R)$ is the moment of inertia of the nucleon 4

$$
\begin{aligned}
\lambda(R) & =\frac{2 \pi}{3} \int r^{2} d r \Lambda(R) \\
\Lambda(R) & =\sin ^{2}(F)\left[F_{\pi}^{2} e^{2 \sigma}+\frac{4}{e^{2}}\left(F^{\prime 2}+\frac{\sin ^{2}(F)}{r^{2}}\right)\right]
\end{aligned}
$$

and $\mathrm{F}$ is the skyrmion profile whose $R$ dependence enters through the scalar field $\sigma$. It turns out, as expected, that the spin-orbit of eq. (23) is quite negligible, due to the $\frac{1}{M_{0}^{2}}$ dependence.

There is another source of spin-orbit interaction. It is due to the transformation of the fields to a rotating frame, essentially the coupling of the baryon current to the $\omega$ field in a rotating nucleus analogous to the isoscalar coupling to the photon. However, the $\omega$ meson coupling to the skyrmion, is not a gauge invariant one and we do not expect the same result as for the magnetic moment of the nucleon. The introduction of a gauge invariant coupling to the skyrmion requires additional terms.

In order to find the spin-orbit potential, we need the mean fields for a streaming nucleus. In this case there arises a spatial component of the $\omega$ meson field. An appropriate approximate ansatz for this component is [2]

$$
\boldsymbol{\omega}=\mathbf{V} \omega_{1}(R)=(\boldsymbol{\Omega} \times \mathbf{R}) \omega_{1}(R)
$$


where $\mathbf{V}$ is the tangential velocity of the nucleus at each $R$ and $\Omega$ the angular velocity. At the same time the nucleon baryon density develops a time dependent piece of the form [18]

$$
\mathbf{B}(\mathbf{u})=\mathbf{S} \times \mathbf{u} \frac{B_{0}(u)}{2 \lambda}
$$

where $B_{0}(u)$ is the static baryon density of the skyrmion.

Inserting eqs.(26,27) above in the lagrangian (1), there will appear new terms in energy. After averaging over the angular directions of $\mathbf{R}$, we find the equation of motion for $\omega_{1}$ to be

$$
\omega_{1}^{\prime \prime}+\frac{4 \omega_{1}^{\prime}}{R}-m_{\omega}^{2} \omega_{1} e^{2 \sigma}+g_{V} B=0
$$

This is very similar to the equation of motion of the static $\omega$ in eq. (18). We solve equation (28) for each nucleus using the scalar field of the static case and demanding a vanishing $\omega_{1}$ at infinity.

In order to find the corresponding spin-orbit interaction we consider a nucleon spinning at rest with a nucleus rotating with a velocity, $-\mathbf{V}(R)$, opposite to the direction of rotation of the nucleon. Using the collective coordinate quantization scheme of eq. (21), and the projection formula of eq. (24) we find

$$
W_{\text {s.o. }}=\frac{-\mathbf{S} \cdot \mathbf{L}}{2 M_{0} \lambda(R)} \omega_{1}(R)
$$

Clearly, $W_{\text {s.o. }}$ is more important than $U_{\text {s.o. }}$ due to the $\frac{1}{M_{0}}$ dependence. It is a pure skyrmion spin-orbit as evidenced by the presence of the moment of inertia in the potential.

In the Dirac type of Walecka models [2], the spin-orbit interaction arises from the coupling of the lower components of the Dirac wave function. In conventional nuclear forces calculations the spin-orbit interaction is found by 
including pion exchange [19]. Here, it arises from the interaction of the rigid rotation of the nucleon with the flow of the mean fields, which is quite a different mechanism.

The static fields contribute to the energy of the nucleus an amount proportional to the baryons rest energy, while the dynamical $\omega_{1}$ contribution originates from the rotational energy. The latter then adds a small correction to the static mean fields and may be ignored.

We now focus on the single skyrmion in the nucleus. Using eq.(8) in the relativistically invariant lagrangian of eq. (1), the classical Hamiltonian of a single skyrmion in motion becomes

$$
H=\sqrt{p^{2}+M^{2}}+g_{V} \omega+W_{\text {s.o. }}+U_{\text {s.o. }}
$$

where $p$ is the nucleon momentum, the conjugated variable to the skyrmion center location $R$. Expanding the square root in eq. (30) to order $p^{2}$ and quantizing the coordinate $R$, we obtain an effective Schrödinger equation for the radial wave function of the skyrmion center with total energy $E$

$$
\left[\frac{\partial^{2}}{\partial R^{2}}+\frac{2 \partial}{R \partial R}-\frac{l(l+1)}{R^{2}}-Q(R)\right] \Psi=0
$$

where

$$
\begin{array}{r}
Q(R)=e^{2 \sigma} M_{0}^{2}-\left(-g_{V} \omega+E-W_{\text {s.o. }}\right)^{2}-Z(R) \\
Z(R) \approx \frac{g_{V}}{2 M(R)}\left[\frac{\partial^{2}}{\partial R^{2}}+\frac{2 \partial}{R \partial R}\right] \omega+2 M_{0} U_{\text {s.o. }}
\end{array}
$$

The central potential entering the calculation of $U_{\text {s.o. }}$ of eq. (23) is given by

$$
V_{C}=\frac{Q_{1}(R)}{2 M_{0}}
$$


where $Q_{1}$ is given by $Q(R)$ of eq.(32), but without the spin-orbit pieces. The energy eigenvalue of the Schrödinger equation $E$ appears inside the operator $Q(R)$.

We have solved the Schrödinger equation (31) for the ground state single particle levels for the magic nuclei $C^{12}, O^{16}$ and $C a^{40}$.

The only free parameters are the values of the scalar and the $\omega$ meson at the center of the nucleus. These parameters determine the chemical potential and the cutoff radius. They are fixed once for all the energy levels in the chosen nucleus. We used the measured $\omega$ meson and nucleon masses and $g_{V}=7.31[16]$.

Table 1 shows the comparison between the predicted binding energies and the experimental ones averaged over proton and neutron states[20, 21]. 
Table 1: Binding energies of single particle levels

\begin{tabular}{|c|c|c|c|}
\hline Nucleus & Shell & $\begin{array}{c}\text { calculated energy } \\
\mathrm{MeV}\end{array}$ & $\begin{array}{c}\text { experimental energy } \\
\mathrm{MeV}\end{array}$ \\
\hline \multirow{3}{*}{$C^{12}$} & $1 s_{\frac{1}{2}}$ & 36.3 & 35.2 \\
& $1 p_{\frac{3}{2}}$ & 15.7 & 16.9 \\
\hline \multirow{3}{*}{$O^{16}$} & $1 s_{\frac{1}{2}}$ & 37.1 & $43 \pm 5$ \\
& $1 p_{\frac{3}{2}}$ & 20.5 & 20.1 \\
& $1 p_{\frac{1}{2}}$ & 15.6 & 13.9 \\
& & & \\
\hline \multirow{3}{*}{$a^{40}$} & $1 s_{\frac{1}{2}}$ & 48 & $50 \pm 10$ \\
& $1 p_{\frac{3}{2}}$ & 35 & $34 \pm 6$ \\
& $1 p_{\frac{1}{2}}$ & 30.7 & $34 \pm 6$ \\
& $1 d_{\frac{5}{2}}$ & 21 & 18.5 \\
& $2 s_{\frac{1}{2}}$ & 15.7 & 14.5 \\
& $1 d_{\frac{3}{2}}$ & 14.8 & \\
& & & \\
\hline
\end{tabular}


The results show that the skyrmion picture of both the central and the spin-orbit interaction is quite good. The spin-orbit originates solely from the $\omega$ meson as viewed by the rotating skyrmion, in contradistinction to the Dirac mean field case in which both scalar and vector fields act together to produce a large interaction. It might appear at first that the skyrmion viewed as based upon a $\frac{1}{N_{c}}$ expansion of QCD, cannot lead reliably to such fine detalis such as binding energies of around $20 \mathrm{MeV}$. However, the binding energies we find are not clearly related to the $\frac{1}{N_{c}}$ expansion. The $\frac{1}{N_{c}}$ approximation refers to the properties of the soliton constructed from colored quarks. Finite $N_{c}$ corrections, as well as quantum effects may indeed have influence on the basic properties of the soliton. However, the interaction of the skyrmion with the external scalar and vector fields operating through location of its center, promoted to the rank of quantum collective coordinate, is expected to be relatively unaffected; provided we use the appropriate parameters for the nucleon, namely its mass and moment of inertia.

In the Skyrme model, the nucleon interacts as a colorless object with its neighbors. The 'exchanged' meson fields are also colorless. Whether nucleons are partially deconfined inside the nucleus and quarks can percolate and be exchanged between nucleons, remains an open question. Nevertheless, in the language of skyrmeons, the leading contribution to the nucleon-nucleon interaction is indeed due to processes that ignore the color structure of the baryons, it is color-blind. This assumptions are based on the fact that the interactions between nucleons play a role in nuclear structure, only for internucleon separations that are too large for color forces to be noticed. The effect of those forces is introduced phenomenologically in the parameters of the model, whose scale is not calculated. In some sense, the skyrmion interactions are like Van der Waals forces that act between neutral atoms and molecules. Although their origin resides within electromagnetic interactions, once the effective forces are determined one can work with them. The approximation is even better for quarks because they are confined. 


\section{Nucleon properties in the medium}

In the previous sections we took the value of the meson fields as constant throughout the skyrmion. This method yields the scaling law of eq. (12). In this section we check the validity of this approximation and look for influences of the skyrmion interior on the meson fields and viceversa. Consider now the $\sigma$ and $\omega$ fields radially symmetric, but allowed to change inside the skyrmion, namely

$$
\begin{array}{r}
\sigma=\sigma(z) \\
\omega_{0}=\omega_{0}(z)
\end{array}
$$

where $z$ is the distance from the center of the nucleus. Inside a skyrmion we have

$$
z=\sqrt{R^{2}+r^{2}-2 R r \cos \left(\theta_{R}-\theta_{r}\right)}
$$

with $R$ the center of mass location of the skyrmion and $\mathrm{r}$ the radial distance from this center.

We will simplify the calculation by averaging the fields over angles. The distribution function we use is independent of angle (eq. (13)). The soliton profile will still be taken as spherically symmetric. We could allow for distortions of the nucleon, but this will make the calculation extremely cumbersome. We want to keep the description as simple as possible and still capture the esence of the effects of the meson fields on the skyrmion.

We average over angles, without determining the meson fields yet

$$
Q(r, R)=\frac{1}{(4 \pi)^{2}} \int d \Omega_{R} \int d \Omega_{r} \chi(z)
$$




$$
P(r, R)=\frac{1}{(4 \pi)^{2}} \int d \Omega_{R} \int d \Omega_{r} \omega(z)
$$

With $\chi=e^{2 \sigma}$ obtaining

$$
Q(r, R)=\frac{1}{4 r R} \int_{|R-r|}^{R+r} \chi(u) u d u
$$

with a similar equation for $P$.

The single skyrmion static energy is now

$$
E=4 \pi \int r^{2} d r\left(Q(r, R) M(r)-P(r, R) g_{V} \frac{\sin ^{2} F F^{\prime}}{2 \pi^{2} r^{2}}\right)
$$

where $M(r)$ is defined in eq. (10). The meson fields depend in a nontrivial way on $\mathrm{R}$ and the skyrmion inherits this dependence.

The skyrmion equation of motion becomes

$$
\begin{aligned}
\left(Q+\frac{8 \sin ^{2} F}{\tilde{r}^{2}}\right) F^{\prime \prime} & +F^{\prime}\left(2 Q / r+Q^{\prime}\right)+\frac{4 \sin 2 F F^{2}}{\tilde{r}^{2}}-\frac{Q \sin 2 F}{r^{2}} \\
& -\frac{4 \sin ^{2} F \sin 2 F}{r^{2} \tilde{r}^{2}}-\frac{2 g_{V} P^{\prime} \sin ^{2} F}{\pi^{2} r^{2}}=0
\end{aligned}
$$

where $\tilde{r}=e F_{\pi} r$ and primes denote derivatives with respect to $\mathrm{r}$.

We start the calculation by evaluating the meson fields for the chosen nuclei. The values of the meson fields at the center of the nucleus are varied in order to fit the nuclear densities. We do not parametrize the scalar potential in a definite manner. We take the measured densities as input and solve the Thomas-Fermi equations of motion for the $\omega$ meson with a fixed chemical potential $\mu$. The meson fields are then fitted with analytical expressions that reproduce accurately the calculated values. For the nuclei $C^{12}$ and $O^{16}$ we use formulae that are easily integrable in order to find $Q$ and $P$ of eq. (36) 


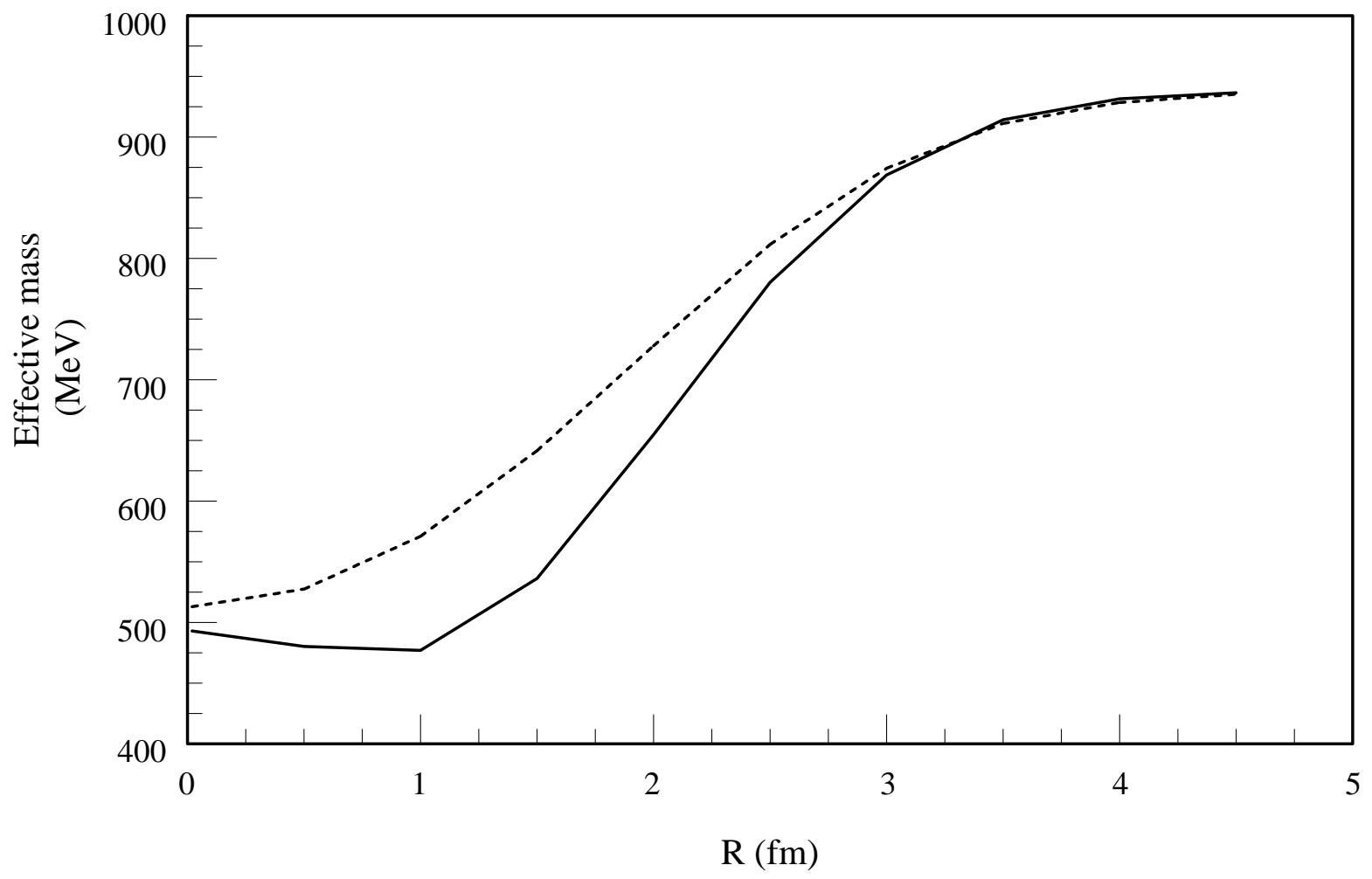

Figure 1: $M_{\text {eff }}$ in $C^{12}$, constant (modified) meson fields inside the skyrmion, full line (dashed line)

$$
\begin{array}{r}
\chi(z)=1+\left(a_{1}+b_{1} z^{2}+c_{1} z^{4}\right) e^{-d_{1} z^{2}} \\
\omega(z)=\left(a_{2}+b_{2} z^{2}+c_{2} z^{4}\right) e^{-d_{2} z^{2}}
\end{array}
$$

whereas for, $C a^{40}$, it was found after trial and error that a better suited expression for the $\omega$ field is

$$
\omega(z)=\left(a_{2}+b_{2} z^{2}+c_{2} z^{4}\right) e^{-d_{2} z}
$$




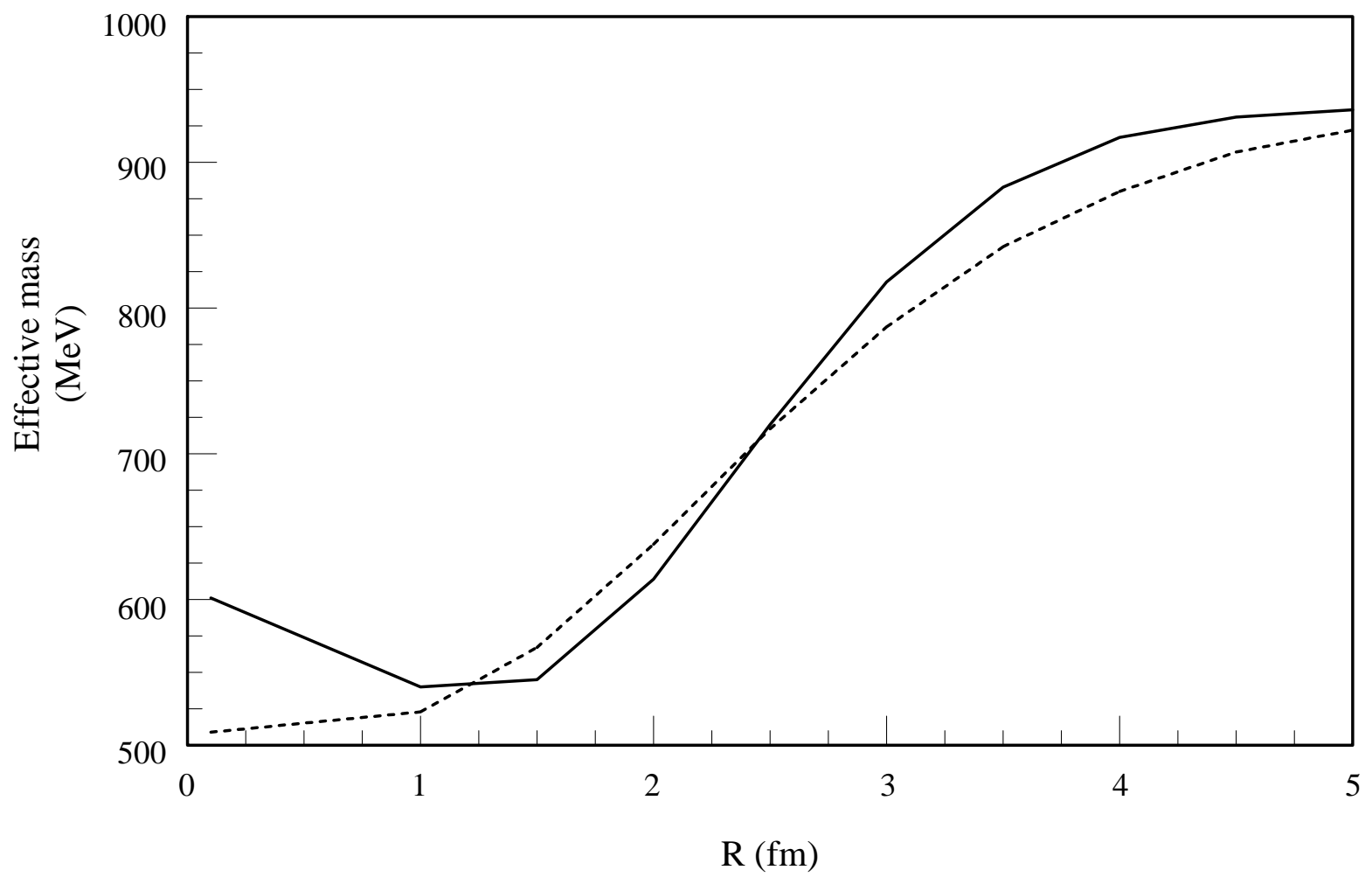

Figure 2: $M_{\text {eff }}$ in $O^{16}$, constant (modified) meson fields inside the skyrmion, full line (dashed line)

We then calculate the new soliton profiles using eq.(39), evaluate the skyrmion effective mass using eq. (38) and compare it to the desired effective mass needed in order to fit the single particle levels obtained in the previous section as good as possible. We iterate the procedure until the effective masses are the closest to the desired values.

Table 2 shows the parameters for the analytical expressions of eqs. (40,41).

Figures 1 through 3 compare the obtained effective skyrmion masses both 


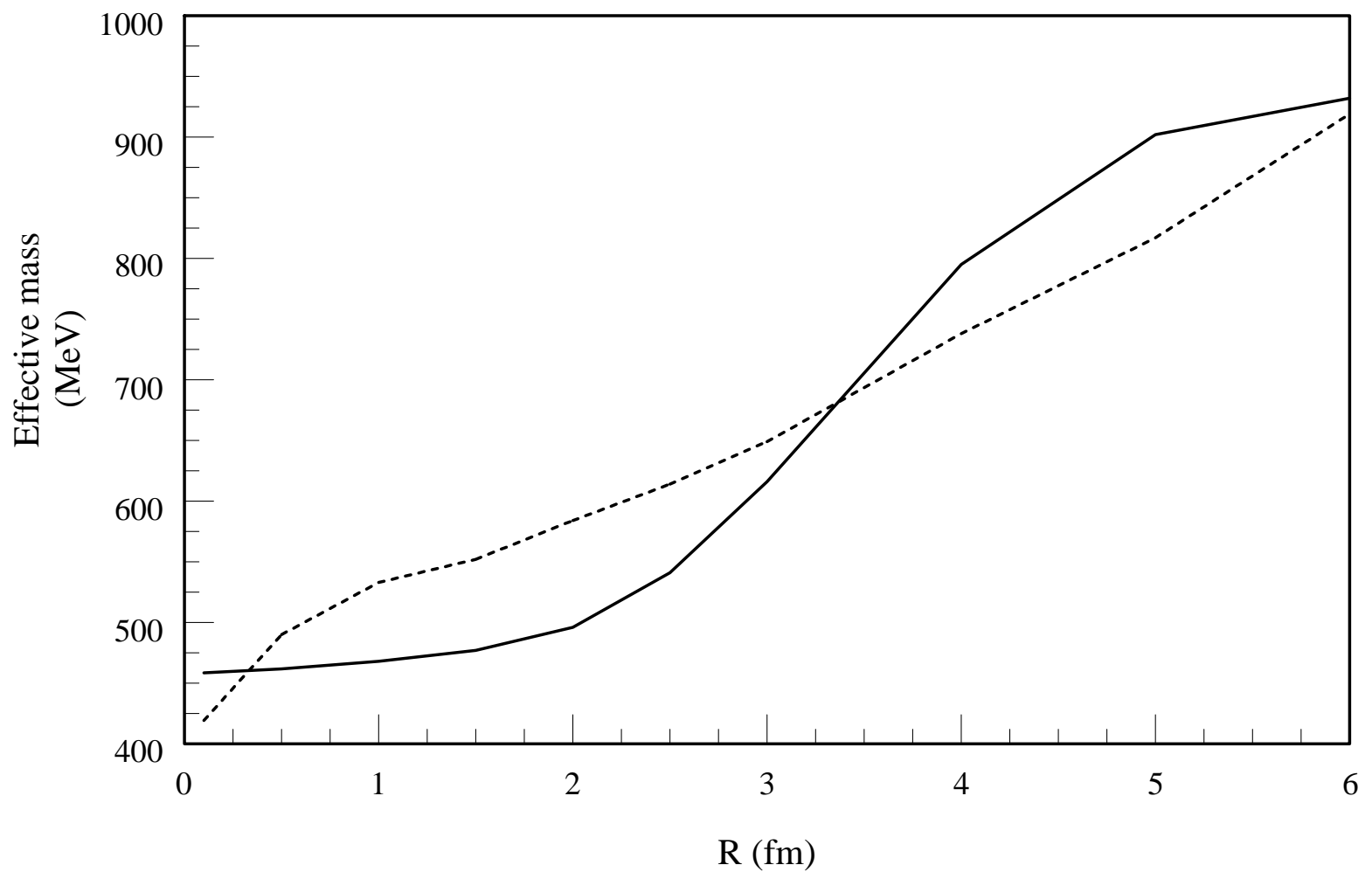

Figure 3: $\quad M_{\text {eff }}$ in $\mathrm{Ca}^{40}$, constant (modified) meson fields inside the skyrmion, full line (dashed line)

with and without meson fields variation inside the skyrmion.

The effective mass of the skyrmion is defined as $M_{0} e^{\sigma_{0}}$ for the case of a constant scalar field inside the nucleon, and

$$
M_{e f f}=E+\frac{3}{8 \lambda}
$$

where $\mathrm{E}$ is the nucleon energy obtained from eq. (38) and $\lambda$ is defined in eq. (25). The trend and absolute values of both types of effective masses are 
Table 2: Parameters for the fits to the meson fields with the analytical expressions of eqs.[42,43]

\begin{tabular}{|c|c|c|c|}
\hline parameter & $C^{12}$ & $O^{16}$ & $C a^{40}$ \\
\hline & & & \\
$a_{1}$ & -0.4744275 & -0.3581017 & -0.5112333 \\
$b_{1}$ & -0.2732692 & -0.2322270 & -0.1049903 \\
$c_{1}$ & $-7.1264720 \mathrm{E}-03$ & $-2.4487255 \mathrm{E}-03$ & $-1.7629359 \mathrm{E}-02$ \\
$d_{1}$ & 0.4292937 & 0.3366868 & 0.2366882 \\
$a_{2}$ & 0.1471073 & 0.1004175 & 0.1679764 \\
$b_{2}$ & $5.3953879 \mathrm{E}-02$ & $5.9962720 \mathrm{E}-02$ & 0.2383440 \\
$c_{2}$ & 0.1121303 & $2.7225710 \mathrm{E}-02$ & $-9.0451920 \mathrm{E}-03$ \\
$d_{2}$ & 0.8262799 & 0.5333008 & 0.9995874 \\
\hline
\end{tabular}

consistent. However, they are not identical. It was impossible to improve the agreement beyond the one shown in the graphs. The inclusion of the volume of the skyrmion in the evaluation of the meson fields cannot be disregarded. This is more clearly seen in figure 4 , where we show the scalar field $\chi=e^{2 \sigma}$ in the nucleus with and without the influence of the volume of the skyrmion for $O^{16}$. A similar picture emerges for the $\omega$ field and in the other nuclei.

Another dramatic effect shows up in the isoscalar root mean square radius of the nucleon. Figure 5 depicts this radius as a function of the location of the center of the skyrmion in $O^{16}$. The nucleon swells inside the nucleus to almost twice its free size. This is a bit troubling, because it would imply a breakdown of the fluid approximation. The nucleons no longer act as free molecules and the solitons overlap. However, the overall picture does not change because the strength of the interaction between solitons dimishes accordingly. A measure of that interaction is the $\pi \mathrm{NN}$ coupling constant [5]. A better estimate of the importance of the overlap between skyrmions will demand a calculation of the skyrmion-skyrmion interaction, but we limit 


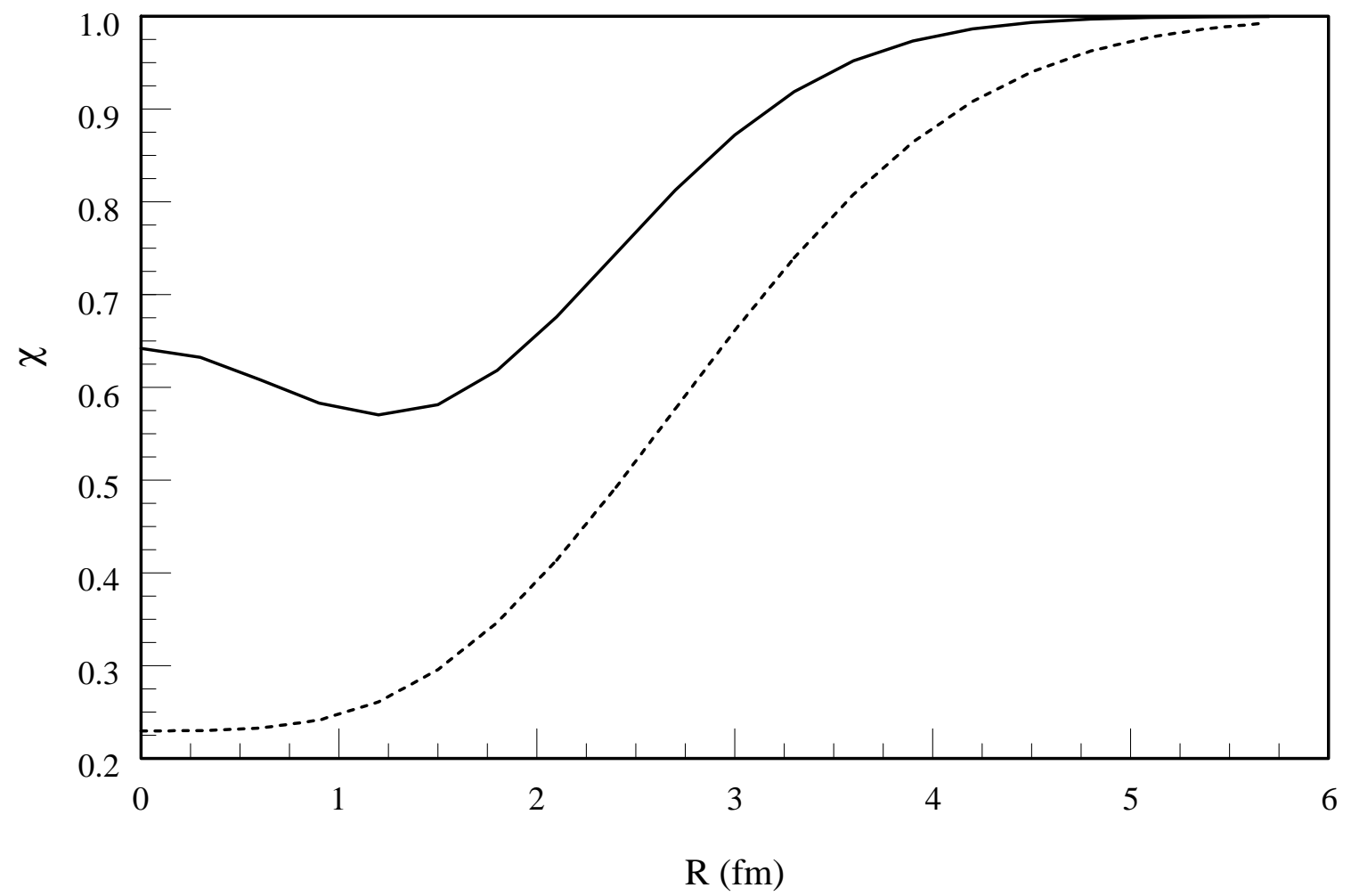

Figure 4: $\quad \chi$ as a function of $R$ in $O^{16}$ for constant (modified) meson fields inside the skyrmion, full line (dashed line)

ourselves to obtain a rough estimate of the effect.

Figure 6 shows this constant as a function of distance for $O^{16}$. It has the very opposite behavior. It drops to almost half its value inside the nucleus. So, perhaps there is still room to consider the nucleons as essentially free objects. Despite the fact that they overlap, the strength of the mutual interaction renders this overlap quite ineffective.

Other nucleon observables, such as magnetic g-factors, axial coupling constant and magnetic radii are less susceptible to changes in the environment. 


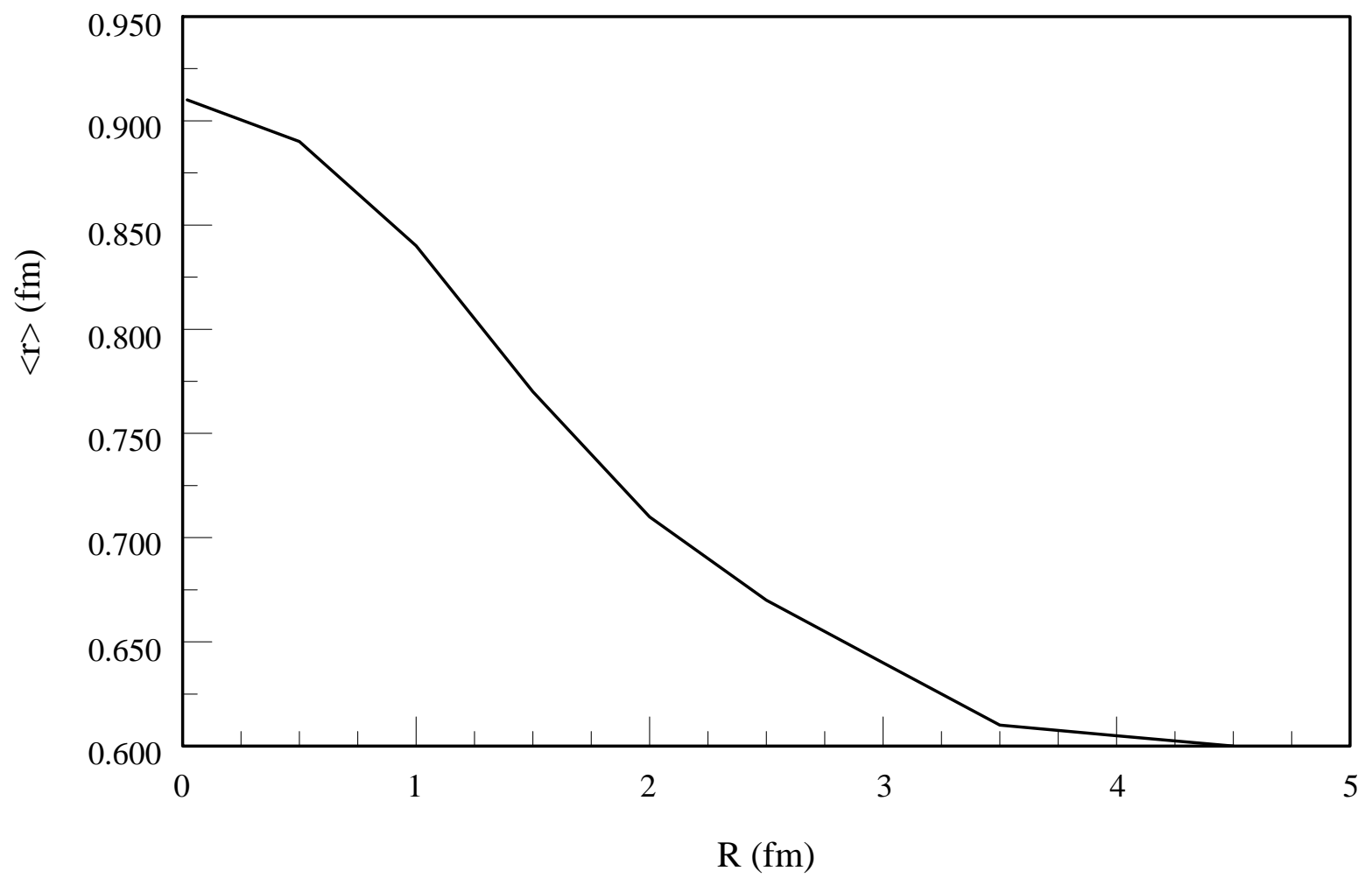

Figure 5: Skyrmion rms radius in $O^{16}$ with modified meson fields

We have attempted to put the scalar fields we found in correspondence with the Kisslinger type of potential with the identification [22], [23].

$$
\begin{gathered}
\chi=\frac{-\kappa}{1+g_{0}^{\prime} \kappa} \\
\kappa=4 \pi c_{0} \rho(R)
\end{gathered}
$$

where $\rho$ is the nuclear density, $c_{0} \approx .21 m_{\pi}^{-3}$, and $g_{0}^{\prime}<1$. This procedure would predict the values of these parameters as suggested by the Skyrme model. Although it was possible to fit the scalar field with the above potential 


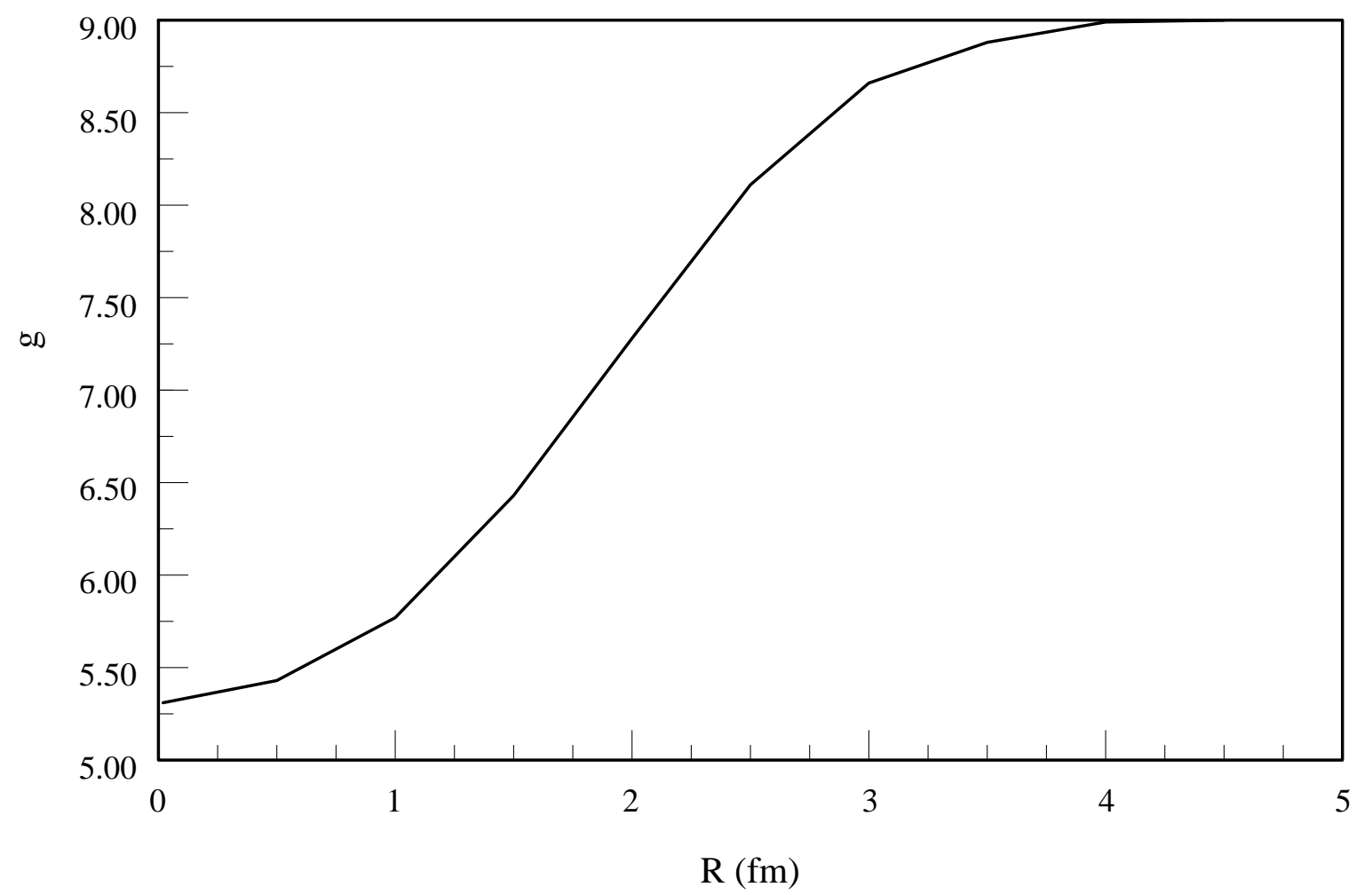

Figure 6: $g_{\pi N N}$ in $O^{16}$ with modified meson fields

around the surface of the nucleus, it is impossible to obtain a moderately reasonable fit inside the nucleus. In all cases there seems to be convergence to a set of parameters that indicate a much larger Landau parameter than expected, approximately around $g_{0}^{\prime} \approx 1$. The Skyrme model appears to be suggesting a strong medium correction to the so-called effective diffraction index. Inside the nucleus nonlinearities play a dominant role. 


\section{Acknowledgements}

This work was supported in part by the Department of Energy under grant DE-FG03-93ER40773 and by the National Science Foundation under grant PHY-9413872.

\section{References}

[1] M. A. Preston and R. K. Bhaduri, Structure of the nucleus AddisonWesley Pub. Co., Advanced Book Program, 1975.

[2] B. D. Serot and J. D. Walecka, Adv. Nucl. Phys. 16 (1986) 1.

[3] T.H.R. Skyrme, Proc. Roy. Soc. London, A260 (1961) 127; A262 (1961) 237 and Nucl. Phys. 31 (1962) 556.

[4] E. Witten, Nucl. Phys. B223 (1983) 422, 433.

[5] G.S. Adkins, C.R. Nappi, and E. Witten, Nucl. Phys. B228 (1983) 552.

[6] J. M. Eisenberg and G. Kälbermann, Nuclear forces from Skyrmions, Int. J. Mod. Phys. E5 (1996) 423.

[7] C. J. Houghton, N. S. Manton and P. M. Sutcliffe, Nucl. Phys. B510 (1998) 507.

[8] T. Ioannidou, B. Piette and W.J. Zakrzewski, hep-th 9808066.

[9] P. Irwin, hep-th 9804142.

[10] J. Schechter, Phys. Rev. D21 (1980) 3393.

[11] G. W. Carter, P. J. Ellis and S. Rudaz, Nucl. Phys. A 618 (1997) 317.

[12] J. Ellis, Nucl. Phys. B22 (1970), 478, and references therein. 
[13] H. Yabu, B. Schwessinger and G. Holzwarth, Phys. Let. B224, 25 (1989).

[14] X. Ji, Phys. Lett. B254 (1990) 456.

[15] G. Kälbermann, Nucl. Phys. A612 (1997) 359.

[16] G. Kälbermann, Nucl. Phys. A633 (1998) 331.

[17] J. D. Jackson, Classical Electrodynamics, John Wiley and sons, 1965, chapter 11 .

[18] D. O. Riska and B. Schwesinger, Phys. Lett. B229 (1989) 339.

[19] S. C. Pieper and V. R. Phandaripande, Phys. Rev. Lett. 70 (1993)2541.

[20] C. Fuchs, H. Lenske and H. H. Wolter, Phys. Rev. C52 (1995) 3043.

[21] G. Landaud et al., Nucl. Phys. A173 (1971) 337.

[22] J.M. Eisenberg and D.S. Koltun, Theory of meson interactions with nuclei (Wiley-Interscience, New York, 1980).

[23] T. Ericson and W. Weise, Pions and nuclei (Oxford University Press, Oxford, 1988). 